IGCS19-0140

\section{A PROSPECTIVE RANDOMISED STUDY OF OPEN VERSUS ROBOTIC ASSISTED PARA AORTIC LYMPH NODE DISSECTION IN HIGH RISK ENDOMETRIAL CARCINOMA - A NOVEL TECHNIQUE}

${ }^{1}$ VK Ahuja*, ${ }^{2} \mathrm{~S}$ Somashekhar, ${ }^{2} \mathrm{~K}$ Ashwin, ${ }^{2} \mathrm{C}$ Rohit Kumar, ${ }^{2} \mathrm{Y}$ Ramya. ${ }^{1}$ Manipal Comphrensive Cancer Centre- Manipal Hospital- Bengaluru, Gynec-oncology, Bengaluru, India; ${ }^{2}$ Manipal Comphrensive Cancer Centre- Manipal Hospital- Bengaluru, Surgical Oncology, Bengaluru, India

10.1136/ijgc-2019-IGCS.54

Objectives To evaluate the technical feasibility and safety of Robotic assisted Para aortic lymphadenectomy in comparison with Open surgery in terms of adequacy of staging, blood loss,lymph node harvest, hospital stay and complications.

Methods A randomized prospective study was performed which included 180 patients diagnosed with endometrial carcinoma who were divided into two groups one open and other robotic. All patients underwent Type I Pan Hysterectomy + B/ L Pelvic lymphadenectomy. The high risk patients (FIGO grade 3 , Tumor $>2 \mathrm{~cm}$, pelvic node positive and $>50 \%$ myoinvasion) were taken up for para aortic lymphadenectomy. The Para-Aortic node dissection was performedupto renal veins. The split and roll technique was used to perform the pre caval and pre aortic lymphadenectomy.

Results Of 180 patients, 113 had high risk endometrial cancer (open 58 and Robotic 55). The average blood loss in open arm was $134.6 \mathrm{ml}$ vs $41.2 \mathrm{ml}$ in robotic arm. In open surgery on average 11.6 nodes were harvested when compared to 17.5 nodes in robotic arm. Duration of hospital stay for open group was 5.54 days vs 1.94 days for robotic arm. None of the patients in either arm had any major intra-operative or post-operative complications. 23 patients in the open arm had prolonged ileus while 4 patients had ileus in robotic arm. 7 patients in open arm developed wound infection.

Conclusions This study showed that robotic assisted paraaortic lymphadenectomy had equal oncologic outcome as compared to open technique. Minimal blood loss and less pain helped in shorter hospital stay and early return to normal activities.

\section{IGCS19-0490}

\section{INFECTIOUS MORBIDITY AFTER SURGERY IN ADVANCED OVARIAN CANCER: CHALLENGES WITH ANTIMICROBIAL RESISTANCE IN A TERTIARY CANCER CENTER IN EASTERN INDIA}

R Shrestha, K Saujanya, M Karar, B Kar, A Mukhopadhyay, A Mukhopadhyay*. Tata Medical Center, Gynaecological Oncology, Kolkata, India

10.1136/ijgc-2019-IGCS.55

Objectives To assess the incidence of postoperative infectious morbidity (POIM) after cytoreductive surgery (CRS) in advanced ovarian cancer (AOC) patients and utilization of pre-operative stool surveillance culture in prediction of antimicrobial resistance (AMR) and treatment of POIM.
Methods Retrospective observational study in FIGO stage III/ IV AOC patients operated between 2015 to 2018 at Tata Medical Center, Kolkata, India. Data was extracted from hospital electronical medical records.

Results Out of 328 patients operated, pre-operative stool culture report was available in 225 women. Multidrug resistant organisms (MDRO) was isolated in 177 (78.6\%); E Coli $(n=136)$, Klebsiella $(n=48)$, Enterobacter $(n=40)$, Psedomonas $(n=2)$, Acinetobactor $(n=1)$. E.Coli was the major organism isolated in blood stream, urine and body fluid in patients with POIM (60\%). Clinical/Microbial confirmed POIM was diagnosed in $107 / 225$ (47.5\%) women up to 30 days post-operatively; Clavien-Dindo(CD)2 in $88, \mathrm{CD} 3$ in $12, \mathrm{CD} 4$ in 5 and CD5 in 2 women. AMR pattern in MDRO: Amoxiclavulanate (90\%), Piperacillin-Tazobactam(50-60\%), Meropenem (3040\%) and Colisitn and Fosfomycin (0-5\%) making Meropenem the most commonly prescribed antibiotic for POIM. In multivariate analysis, Diabetes Mellitus (OR 3.0,CI 1.3-7.0) and Bowel resection (OR4.4, CI 2.2-9.8) were independent risk factors for $\operatorname{POIM}(\mathrm{p}<0.05)$, while splenectomy and diaphragmatic surgery was significantly associated in univariate analysis.

Conclusions CRS in AOC was associated with high incidence of POIM at our setting; pre-operative surveillance stool culture could guide us in starting the appropriate antibiotic postoperatively at the earliest clinical suspicion based on the MDRO profile. Although, the treatment cost is high, > $80 \%$ women go home around the 7 th post-operative day.

\section{IGCS19-0491}

\section{FIRST INDIAN STUDY ON PRESSURIZED INTRAPERITONEAL AEROSOL CHEMOTHERAPY (PIPAC) FOR ADVANCED PERITONEAL CARCINOMATOSIS SECONDARY TO EPITHELIAL OVARIAN CANCER AND ITS IMPACT ON QUALITY OF LIFE}

${ }^{1} \mathrm{~S}$ Somashekar*, ${ }^{1} \mathrm{~K}$ Ashwin, ${ }^{1} \mathrm{C}$ Rohit Kumar, ${ }^{1} \mathrm{~A}$ Vijay Kumar, ${ }^{2} \mathrm{~A}$ rauthan, ${ }^{1} \mathrm{Y}$ Ramya. ${ }^{1}$ Manipal Comphrensive Cancer Centre, Surgical Oncology, Bengaluru, India; ${ }^{2}$ Manipal Comphrensive Cancer Centre, Medical Oncology, Bengaluru, India

\subsection{6/ijgc-2019-IGCS.56}

Objectives Peritoneal carcinomatosis is a common evolution in ovarian cancers. Although majority of patients have option of cytoreductive surgery and HIPEC, a few with recurrent cancers who are not eligible for curative approach can undergo pressurized intraperitoneal aerosol chemotherapy (PIPAC). It is a safe and innovative approach, which enhances the effect of intraperitoneal delivery of chemotherapy without major toxicity.

Methods Between June 2017 and December 2018, 35 PIPAC applications were done in 15 patients using cisplatin and doxorubicin. The patient demographics, perioperative findings, adverse events, and outcomes were prospectively recorded.

Results 35 PIPAC administrations were performed in 15 patients with peritoneal carcinomatosis secondary to ovarian cancer. The median hospital stay was 1.5 day. 10 patients completed all 3 pipac. One patient had bowel perforation; one had major bleeding during the procedure. CTCAE 
grade 2 was observed in 7 patients, for abdominal pain and nausea. C- reactive protein was elevated in all patients, renal and hepatic functions were not impaired in any patients. Of the 15 patients, 9 patients had partial response, 4 had stable disease \& 2 had complete response. The global physical score deteriorated slightly after 1st PIPAC (from $84 \%$ to $71 \%$ ), but improved after PIPAC \# 2 (up to $88 \%$ ). Gastrointestinal symptoms \& pain score remained stable under PIPAC therapy.

Conclusions Our results show the feasibility and safety of PIPAC for patients with advanced ovarian cancer. The procedure has low morbidity with no mortality \& short learning curve. There was no therapy related deterioration of quality of life after PIPAC.

\section{IGCS19-0163}

\section{WEIGHT PERCEPTION AND SOCIODEMOGRAPHIC CHARACTERISTICS FOLLOWING ENDOMETRIAL CANCER TREATMENT}

${ }^{1} \mathrm{M}$ Janda*, ${ }^{2} \mathrm{~V}$ Gebski, ${ }^{3} \mathrm{P}$ Forder, ${ }^{4} \mathrm{~A}$ Obermair. 'University of Queensland, Centre for Health Services Research- Faculty of Medicine, Brisbane, Australia; ${ }^{2}$ University of Sydney, NHMRC Clinical Trials Centre, Sydney, Australia; ${ }^{3}$ University of Newcastle, Research Centre for Generational Health and Ageing- Faculty Health and Medicine, Newcastle, Australia; ${ }^{4}$ The University of Queensland, Faculty of Medicine- Centre for Clinical Research, Brisbane, Australia

10.1136/ijgc-2019-IGCS.57

Objectives To explore the association between self-reported 'unhappiness with weight' and sociodemographic characteristics and weight control behaviors among women who underwent hysterectomy for Stage I endometrial cancer.

Methods Women diagnosed with Stage I endometrial cancer who participated in the Laparoscopic Approach to Cancer of the Endometrium (LACE) trial were invited to complete a five-year follow up survey to evaluate their health status, lifestyle and behaviors including their weight perception and use of weight control methods. Of the 516 eligible patients, 259 $(50.2 \%)$ agreed to participate in the survey.

Results At follow-up, women who self-reported they were unhappy with their weight were significantly more likely to have an annual income >AUD 40,000 (ORadjusted 2.7; $\mathrm{p}=0.025)$. Women who were unhappy with their weight were more likely to be younger at follow-up compared to women who were happy with their weight (ORadjusted 0.94; $p=0.003)$. Weight loss programs completed in the twelve months prior to completing the survey were strongly predictive of unhappiness with weight; including exercise (ORadjusted $6.3 ; \mathrm{p}<0.001)$, reduced meal intake (OR 5.2; $\mathrm{p}<0.001$ ) and reduced fats/sugar intake (OR 5.4; $\mathrm{p}<0.001)$. Ever-use of commercial programs and diets from dietary books were also associated with unhappiness with weight at follow-up $(\mathrm{p} \leq 0.03)$.

Conclusions Our study provides evidence that many women continue to be unhappy with weight many years after their endometrial cancer treatment. Supporting their self-directed efforts to lose weight may increase benefit of available programs, and therefore women's satisfaction with current weight.

\section{Europe Regional Plenary IGCS19-0510}

\section{SYNDECAN-1 INHIBITION REVERSES THE PRE- MALIGNANT PHENOTYPE OF ENDOMETRIOMA THROUGH TGF-BETA SIGNALLING: POTENTIAL IMPLICATIONS IN ENDOMETRIOSIS ASSOCIATED OVARIAN CANCER}

\begin{abstract}
${ }^{1} \mathrm{~S}$ Ponandai-Srinivasan*, ${ }^{2} \mathrm{M}$ Saare, ${ }^{1} \mathrm{NR}$ Boggavarapu, ${ }^{3} \mathrm{~S}$ Ehström, ${ }^{1} \mathrm{PA}$ Garcia-Uribe ${ }^{2,4} \mathrm{~A}$ Salumets, ${ }^{5} \mathrm{M}$ Götte, ${ }^{1} \mathrm{PG}$ Lalitkumar, ${ }^{1} \mathrm{~K}$ Gemzell-Danielsson. ${ }^{1}$ Karolinska Institute, Department of Women's and Children's health, Stockholm, Sweden; 'University of Tartu, Department of Obstetrics and Gynecology, Tartu, Estonia; ${ }^{3}$ Sofiahemmet Hospital, Ultragyn Stockholm, Sweden: ${ }^{4}$ University of Helsinki and Helsinki University Hospital, Department of Obstetrics and Gynaecology, Helsinki, Finland; ${ }^{5}$ Muenster University- Medical Center Department of Gynecology and Obstetrics, Meunster, Germany
\end{abstract}

\subsection{6/ijgc-2019-IGCS.58}

Objectives Transforming growth factor-beta (TGF- $\beta$ ) is abundantly expressed in peritoneal fluid and endometrioma of women with endometriosis. Similarly, transmembrane proteoglycans of the Syndecan family (SDC), act as co-receptors for growth signalling factors and are aberrantly expressed in endometriotic tissues. Here, we aim to investigate the regulation of SDC-1 upon induced activation with TGF- $\beta$ in vitro, to better understand their interactions and involvement in the pathophysiology of endometriosis.

Methods Endometrioma biopsies $(n=15)$ were obtained from women diagnosed with endometriosis and not received any hormonal treatment. Tissue biospies were investigated for intra-patient heterogeneity using pre-validated panel of stemand cancer- cell signalling genes. Simultaneously, patientderived endometriotic stem/stromal cells $\left(\mathrm{CD} 90^{+}\right.$ $\mathrm{CD}_{73}{ }^{+} \mathrm{CD} 105^{+}, \mathrm{SC}^{+}$) were allowed to generate $3 \mathrm{D}$-spheroids in absence or presence of rhTGF- $\beta$ or TGFBRI/II inhibitor Ly2109761 in vitro; assessed for its influence on SDC-1 expression, proliferation and invasive behaviour. Further, transcriptomic signatures after 3D-spheroid invasion was evaluated upon combining SDC-1 gene silencing with rhTGF- $\beta$ treatment.

Results Clustering analysis from endometriotic tissue gene expression revealed in 2/15 samples (referred to as Endo-hi) aberrant expression of molecules of TGF- $\beta$ signalling (TGF- $\beta 1$, ESR1, CTNNB1, SNAI1, BMI1) which grouped separately from low expression samples (Endo-lo) by $>95 \%$ CI. 3Dspheroids from Endo-hi SC+ exposed to rhTGF- $\beta$ treatment showed increased SDC-1 expression and higher 3D-spheroid invasion compared to Endo-lo SC+. However, rhTGF $\beta$ treatment following SDC-1 gene silencing reversed the higher 3Dinvasion potential and exhibited downregulation of cancer associated pathways.

Conclusions Modulation of SDC- 1 reverses the pre-malignant phenotype of endometrioma and may reduce the potential risk for endometriosis associated ovarian cancer. 